\title{
Discrete Frequency and Phase Coding Waveform for MIMO Radar
}

\author{
Guanghong CHANG ${ }^{1}$, Xiaoxi $Y U^{2}$, Changjun $Y U^{3}$ \\ ${ }^{1}$ School of Electronics and Information Engineering, Harbin Institute of Technology, \\ Xidazhi Street 92, 150001 Harbin, China \\ ${ }^{2}$ School of Electrical and Electronics Engineering, University of Bristol, \\ Senate House, Tyndall Avenue, BS8 1TH Bristol, UK \\ ${ }^{3}$ School of Information and Electrical Engineering, Harbin Institute of Technology at Weihai, \\ West Wenhua Road 2, 264209 Weihai, China
}

changguanghong@126.com,xy13782@my.bristol.ac.uk,yuchangjun@hit.edu.cn

Submitted November 29, 2016 / Accepted April 10, 2017

\begin{abstract}
In multi-input multi-output (MIMO) radar system, good orthogonality between transmitting waveforms will fairly simplify the signal processing, along with improve the targets detection as well as the parameters estimation performance of the system. In this paper, a discrete frequency and phase coding waveform (DFPCW), which attains good orthogonality by varying the carrier frequency and initial phase of each pulse in the pulse train, is designed. The theoretical derivations of ambiguity function and cross ambiguity function of the DFPCW are also given. After then, a generic algorithm is applied by optimizing the carrier frequency code sequence and initial phase code sequence to minimize both the auto-correlation sidelobe peaks and cross-correlation peaks of the waveforms. The simulation results demonstrate that DFPCW has better orthogonality and sidelobe property compared with the traditional discrete frequency coding waveform and widely employed frequency modulated continuous wave, henceforth this new waveform may become to an alternative option for MIMO radar.
\end{abstract}

\section{Keywords}

MIMO radar, orthogonal waveform design, discrete frequency and phase coding waveform

\section{Introduction}

Distinct waveforms can be emitted by each transmitting antenna of the multi-input multi-output (MIMO) radar individually, giving more freedom to systems during transmission [1], [2]. To fulfill the demand of simpler targets detection and parameter estimating, the orthogonal waveforms transmission has become to a general assumption when analyzing the performance of various MIMO radars as well as processing signals [3-5]. However, in reality, no transmitted waveform is perfectly orthogonal, thus how to producing waveforms with good orthogonality is being explored indepth when designing a MIMO radar waveform [6], [7].
Generally, there are three distinct diversities that can be employed when considering the orthogonality between signals: time diversity, frequency diversity and code diversity [3], [8]. For orthogonal signals employing staggered time or multiple frequencies (including carrier frequencies and Doppler frequencies), the coherence of targets may be decreased. Therefore, achieving good orthogonality with code diversity is preferred in many theoretical analysis and practical MIMO radar systems. In 2004, Deng employed simulated annealing algorithm along with optimized the crosscorrelation of waveforms by means of phase modulation, obtaining a desirable pair of phase coding waveforms possessing the optimal auto-correlation sidelobe peak (ASP) and cross-correlation peak (CP) [9], and improvements are given in [10]. After that, discrete frequency coding waveform (DFCW) optimizing the frequency coding sequence of Costas waveforms, has been studied extensively [11-15]. Replacing the constant frequency with linear frequency modulation in the DFCW is also extensive investigated [16-18]. However, the basis of the above mentioned orthogonal coding waveforms [11-18] depends on frequency diversity advantage, which can only provide limited orthogonality in frequency domain.

In this paper, we find that a new kind of variable can be introduced in the cross ambiguity function by a change of the initial phase of each pulse, offering a new diversity to further decrease the cross-correlation of waveforms. Based on this, we propose discrete frequency and phase coding waveform (DFPCW), which has better orthogonality with the use of genetic algorithm optimizing both the frequency code sequence and initial phase code sequence of the pulse train. This paper is structured as follows. Section 2 presents the derivation of ambiguity function and cross ambiguity function of DFPCW. Taking advantage of genetic algorithm, the optimal designing algorithm of DFPCW is also given. In Sec. 3, we compare the DFPCW with several existing orthogonal waveforms, such as DFCW and frequency modulated continuous wave. 


\section{Discrete Frequency and Phase Cod- ing Waveform}

For a MIMO radar system with $L$ transmitting antennas, the normalized DFPCW signal transmitted by the $l$ th antenna can be expressed as

$$
u^{l}(t)=\frac{1}{\sqrt{N}} \sum_{n=0}^{N-1} u_{n}^{l}\left(t-n T_{\mathrm{r}}\right)
$$

where

$$
u_{n}^{l}(t)=\frac{1}{\sqrt{T}} \operatorname{rect}\left(\frac{t}{T}-\frac{1}{2}\right) \exp \left\{\mathrm{j}\left(2 \pi f_{n}^{l} t+\varphi_{n}^{l}\right)\right\}
$$

where $N$ is the number of pulses included in the pulse trains of the DFPCW, $T_{\mathrm{r}}$ is the pulse repetition interval, $T$ is the pulse width, $\operatorname{rect}(\cdot)$ is the rectangular window, $f_{n}^{l}=f_{\mathrm{c}}+F_{n}^{l} \Delta f$ and $\varphi_{n}^{l}=P_{n}^{l} \Delta \varphi$ represent the frequency and the phase of each pulse, respectively, $f_{\mathrm{c}}$ represents the carrier frequency of the radar system, $\Delta f$ and $\Delta \varphi$ represent the frequency and phase step size. $\left\{F_{n}^{l}=0,1, \cdots, N_{\mathrm{f}}-1\right\}$ and $\left\{P_{n}^{l}=0,1, \cdots, N_{\mathrm{p}}-1\right\}$ represent the frequency and phase coded values, $N_{\mathrm{f}}$ and $N_{\mathrm{p}}$ represent the number of the frequency and phase coding. The frequency and phase diagram of the DFPCW is shown in Fig. 1.

\subsection{Ambiguity Function}

The ambiguity function of the $l$ th transmitting signal is given by [19]

$$
\chi^{l}(\tau, v)=\int_{-\infty}^{+\infty} u^{l}(t) u^{l^{*}}(t+\tau) \exp \{\mathrm{j} 2 \pi v t\} \mathrm{d} t
$$

where $\tau$ and $v$ are the time delay and the Doppler shift, respectively, superscript $*$ denotes the complex conjugate. Replacing $u^{l}(t)$ by (1) we have

$$
\begin{aligned}
\chi^{l}(\tau, v)= & \int_{-\infty}^{+\infty}\left(\frac{1}{\sqrt{N}} \sum_{n=0}^{N-1} u_{n}^{l}\left(t-n T_{\mathrm{r}}\right)\right) \\
& \times\left(\frac{1}{\sqrt{N}} \sum_{m=0}^{N-1} u_{m}^{l}{ }^{*}\left(t+\tau-m T_{\mathrm{r}}\right)\right) \exp \{\mathrm{j} 2 \pi v t\} \mathrm{d} t .
\end{aligned}
$$

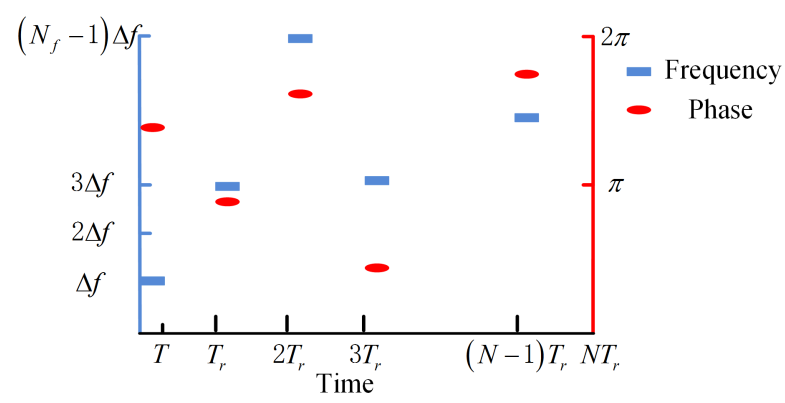

Fig. 1. Frequency and phase diagram of DFPCW. The (blue) retangular represnts the carrier frquency of each pulse, and the (red) ellipse represnts the phase of each pulse.
Substituting (2) in (4) gives

$$
\begin{aligned}
\chi^{l}(\tau, v) \\
=\frac{1}{N T} \int_{-\infty}^{+\infty} \sum_{n=0}^{N-1} \operatorname{rect}\left(\frac{t-n T_{\mathrm{r}}}{T}-\frac{1}{2}\right) \operatorname{rect}\left(\frac{t+\tau-n T_{\mathrm{r}}}{T}-\frac{1}{2}\right) \\
\quad \times \exp \left\{\mathrm{j}\left(2 \pi f_{n}^{l}\left(t-n T_{\mathrm{r}}\right)+\varphi_{n}^{l}\right)\right\} \\
\quad \times \exp \left\{-\mathrm{j}\left(2 \pi f_{n}^{l}\left(t+\tau-n T_{\mathrm{r}}\right)+\varphi_{n}^{l}\right)\right\} \exp \{\mathrm{j} 2 \pi v t\} \mathrm{d} t
\end{aligned}
$$

where $|\tau| \leq T$ and $T \leq T_{\mathrm{r}} / 2$ are assumed. This can be simplified by applying the sum rules of integration. Hence

$$
\begin{aligned}
\chi^{l}(\tau, v) & =\frac{1}{N T} \sum_{n=0}^{N-1} \exp \left\{-\mathrm{j} 2 \pi f_{n}^{l} \tau\right\} \int_{-\infty}^{+\infty} \exp \{\mathrm{j} 2 \pi v t\} \\
& \times \operatorname{rect}\left(\frac{t-n T_{\mathrm{r}}}{T}-\frac{1}{2}\right) \operatorname{rect}\left(\frac{t+\tau-n T_{\mathrm{r}}}{T}-\frac{1}{2}\right) \mathrm{d} t
\end{aligned}
$$

Since the integrand function is the product of two rectangular functions, the bounds of the integral are given by

$$
\begin{aligned}
& \begin{cases}t_{\mathrm{u}}=T-\tau+n T_{\mathrm{r}} & \text { for } \tau \geq 0, \\
t_{\mathrm{d}}=n T_{\mathrm{r}} & \\
\begin{cases}t_{\mathrm{u}}=T+n T_{\mathrm{r}} \\
t_{\mathrm{d}}=-\tau+n T_{\mathrm{r}}\end{cases} & \text { for } \tau<0 .\end{cases}
\end{aligned}
$$

Hence the integral in (6) is solved to give the expression of the ambiguity function of DFPCW

$$
\begin{aligned}
\chi^{l}(\tau, v)= & \sum_{n=0}^{N-1} \exp \left\{-\mathrm{j} 2 \pi f_{n}^{l} \tau\right\} \exp \left\{-\mathrm{j} \pi \nu\left(T+\tau+2 n T_{\mathrm{r}}\right)\right\} \\
& \times \frac{T-|\tau|}{N T} \operatorname{sinc}(v(T-|\tau|)), \quad \text { for }|\tau| \leq T
\end{aligned}
$$

where $\operatorname{sinc}(x)=\sin (\pi x) /(\pi x)$ is the normalized sinc function. The vanished initial phase terms $\varphi_{n}^{l}$ in (8) demonstrate that DFPCW signal has the same ambiguity function with the DFCW. Hence for a given frequency coding sequence, the shape of ambiguity function will remain constant even when the initial phase of the pulse is changed. When the Doppler shift $v=0$, the auto-correlation of DFPCW is

$$
\chi^{l}(\tau, 0)=\frac{T-|\tau|}{N T} \sum_{n=0}^{N-1} \exp \left\{-\mathrm{j} 2 \pi f_{n}^{l} \tau\right\}
$$

which indicates that the auto-correlation of DFPCW depends only on the frequency coding sequence. If the number of the frequencies is the same as that of the pulses, and each of these pulses has unique frequency, then (9) can be simplified as

$$
\chi^{l}(\tau, 0)=\frac{T-|\tau|}{T} \frac{\sin (\pi N \Delta f \tau)}{N \sin (\pi \Delta f \tau)} \exp \{\mathrm{j} \pi(N-1) \Delta f \tau\}
$$

The vanish of frequency coding index $n$ indicates that in this condition the auto-correlation of the DFPCW is independent of the frequency coding sequence. To a certain extent, this property may simplify the optimal design of DFPCW, however, the price paid is the sacrifice of the degrees 
of freedom of the solution. By assuming that the time delay $\tau=0$ in (8), the zero-delay cut is

$$
\begin{aligned}
& \chi^{l}(0, v) \\
& \quad=\operatorname{sinc}(v T) \frac{\sin \left(\pi v N T_{\mathrm{r}}\right)}{N \sin \left(\pi v T_{\mathrm{r}}\right)} \exp \left\{-\mathrm{j} \pi v\left(T+(N-1) T_{\mathrm{r}}\right)\right\} .
\end{aligned}
$$

From (11), we can find that the Doppler resolution and Doppler sidelobe behavior of the DFPCW are independent of the carrier frequency and initial phase. Those Doppler properties relate with the pulse parameter of the waveform, such as pulse width, pulse repetition interval and pulse number. In the following subsection, we will derive the cross ambiguity function of the designed DFPCW.

\subsection{Cross Ambiguity Function}

The cross ambiguity function between the $k$ th and the $l$ th signal transmitted via the transmitting antenna is defined as

$$
\phi^{k l}(\tau, v)=\int_{-\infty}^{+\infty} u^{k}(t) u^{l^{*}}(t+\tau) \exp \{\mathrm{j} 2 \pi v t\} \mathrm{d} t .
$$

Substituting (1) into (12), we have

$$
\begin{aligned}
\phi^{k l}(\tau, v)= & \int_{-\infty}^{+\infty}\left(\frac{1}{\sqrt{N}} \sum_{n=0}^{N-1} u_{n}^{k}\left(t-n T_{\mathrm{r}}\right)\right) \\
& \times\left(\frac{1}{\sqrt{N}} \sum_{m=0}^{N-1} u_{m}^{l^{*}}\left(t+\tau-m T_{\mathrm{r}}\right)\right) \exp \{\mathrm{j} 2 \pi v t\} \mathrm{d} t
\end{aligned}
$$

Assuming $T \leq T_{\mathrm{r}} / 2$ and $|\tau| \leq T$. Recalling (2), we obtain

$$
\begin{aligned}
\phi^{k l}(\tau, v) \\
=\frac{1}{N T} \int_{-\infty}^{+\infty} \sum_{n=0}^{N-1} \operatorname{rect}\left(\frac{t-n T_{\mathrm{r}}}{T}-\frac{1}{2}\right) \operatorname{rect}\left(\frac{t+\tau-n T_{\mathrm{r}}}{T}-\frac{1}{2}\right) \\
\quad \times \exp \left\{\mathrm{j}\left(2 \pi f_{n}^{k}\left(t-n T_{\mathrm{r}}\right)+\varphi_{n}^{k}\right)\right\} \\
\quad \times \exp \left\{-\mathrm{j}\left(2 \pi f_{n}^{l}\left(t+\tau-n T_{\mathrm{r}}\right)+\varphi_{n}^{l}\right)\right\} \exp \{\mathrm{j} 2 \pi v t\} \mathrm{d} t .
\end{aligned}
$$

The sum rules of integration are applied to simplify it

$$
\begin{aligned}
\phi^{k l}(\tau, v)= & \frac{1}{N T} \sum_{n=0}^{N-1} \exp \left\{\mathrm{j} 2 \pi\left(\varphi_{n}^{k l}-f_{n}^{l} \tau-f_{n}^{k l} n T_{\mathrm{r}}\right)\right\} \\
& \times \int_{-\infty}^{+\infty} \exp \left\{\mathrm{j} 2 \pi\left(f_{n}^{k l}+v\right) t\right\} \\
& \times \operatorname{rect}\left(\frac{t-n T_{\mathrm{r}}}{T}-\frac{1}{2}\right) \operatorname{rect}\left(\frac{t+\tau-n T_{\mathrm{r}}}{T}-\frac{1}{2}\right) \mathrm{d} t
\end{aligned}
$$

where $f_{n}^{k l}=\left(F_{n}^{k}-F_{n}^{l}\right) \Delta f, \varphi_{n}^{k l}=\left(P_{n}^{k}-P_{n}^{l}\right) \Delta \varphi$. For the given bounds of the integral shown in (7), the cross ambiguity function can be written as

$$
\begin{aligned}
\phi^{k l}(\tau, v)= & \sum_{n=0}^{N-1} \exp \left\{\mathrm{j} 2 \pi\left(\varphi_{n}^{k l}-f_{n}^{l} \tau-f_{n}^{k l} n T_{\mathrm{r}}\right)\right\} \\
& \times \exp \left\{-\mathrm{j} \pi\left(f_{n}^{k l}+v\right)\left(T+\tau+2 n T_{\mathrm{r}}\right)\right\} \\
& \times \frac{(T-|\tau|)}{N T} \operatorname{sinc}\left(\left(f_{n}^{k l}+v\right)(T-|\tau|)\right) .
\end{aligned}
$$

Therefore, the cross ambiguity function of DFPCW is a function of frequency code sequence and phase code sequence, in particular, when the Doppler shift $v=0$, the cross-correlation of the two waveform is given below

$$
\begin{aligned}
\phi^{k l}(\tau, 0) & \\
= & \frac{(T-|\tau|)}{N T} \sum_{n=0}^{N-1} \exp \left\{\mathrm{j} 2 \pi\left(\varphi_{n}^{k l}-f_{n}^{l} \tau-f_{n}^{k l} n T_{\mathrm{r}}\right)\right\} \\
& \times \exp \left\{-\mathrm{j} \pi f_{n}^{k l}\left(T+\tau+2 n T_{\mathrm{r}}\right)\right\} \operatorname{sinc}\left(f_{n}^{k l}(T-|\tau|)\right)
\end{aligned}
$$

Equation (17) indicates that, the phase difference $\varphi_{n}^{k l}$ as well as frequency difference $f_{n}^{k l}$ of each pulse has influence on the cross-correlation between the two signals. Compared with the traditional DFCW with frequency diversity only, by introducing phase code, additional degrees of freedom during the waveform design are provided, which may be beneficial for the improvement of the waveform performance.

\subsection{Optimal Criteria and Algorithm}

As mentioned in Sec. 2.2, the CP of the transmitting signals is related both to the frequency and phase code sequences. In traditional radar waveform design, low ASP of a waveform is preferred, since the ASP of a strong target may higher than the mainlobe of a weak target. In MIMO radar applications, low cross interference between waveforms may imply better system performance. Thus, we consider to design the DFPCW with optimal ASP and CP. This paper sets the summation of ASP and CP as the fitness function

$$
\begin{aligned}
E= & \sum_{l=0}^{L-1} \operatorname{extr}\left\{\left|\chi^{l}(\tau, 0)\right|, \tau \neq 0\right\} \\
& +w \sum_{k=0}^{L-2} \sum_{l=k+1}^{L-1} \max \left\{\left|\phi^{k l}(\tau, 0)\right|\right\}
\end{aligned}
$$

where extr $\{\cdot\}$ represents extrema, the first term on the right hand side of the equation is the summation of all ASP of waveforms, the second term is the addition of all CP of waveforms, $w$ represents the weight. The setting of $w$ may arbitrary or base on experience. However, in our algorithm, we first generate $N_{\mathrm{g}}$ random waveforms to calculate the average level of the ASP and CP of general DFPCW can achieved. The detailed procedure of the algorithm is shown in Fig. 2. 


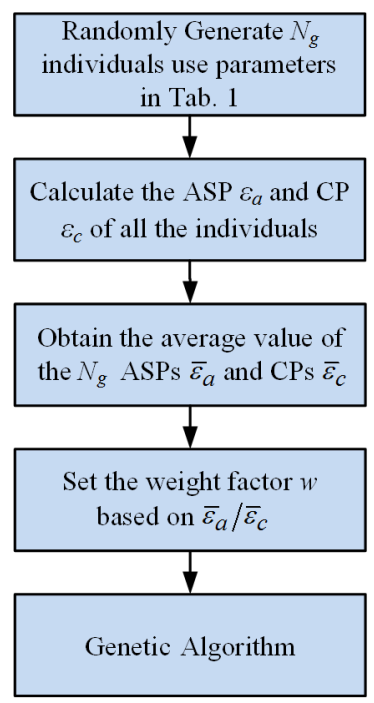

Fig. 2. Flowchart of the algorithm.

\section{Simulations and Comparisons}

In this section, two different methods of simulation are explored to analyze the performance of the DFPCW. The first aims at improving the waveform orthogonality through introducing the phase coding based on existing DFCW, while the second is that the frequency coding as well as phase coding are combined to produce the optimal DFPCW. Comparisons between DFPCW and DFCW are also discussed.

The design method of DFCW has been introduced in [11] thoroughly. Since there are no repeated codes in the sequence, with the ACF of DFCW shown as (10), the sidelobe level cannot be reduced by simply changing the order of the codes. Therefore, we allow the repetition of codes, meanwhile increasing the coding dimension from $N$ ! to $N^{N}$ aims to broadening the search scope of optimal solutions. Moreover, in general, one prefers to optimize CP rather than energy when drawing the optimal DFCW because CP may result in false targets.

This paper brings about the optimal DFCW sequence with genetic algorithm, the weight $w$ in (18) is calculated from $N_{\mathrm{g}}=1000$ random waveform pairs, the simulation parameters are shown in Tab. 1 , and the terminating condition

\begin{tabular}{|l|l|}
\hline Parameters & Values \\
\hline Waveform number $(L)$ & 3 \\
\hline Pulse number $(N)$ & 32 \\
\hline Frequency code number $\left(N_{\mathrm{f}}\right)$ & 32 \\
\hline Phase code number $\left(N_{\mathrm{p}}\right)$ & 32 \\
\hline Carrier frequency $\left(f_{\mathrm{c}}\right)$ & $4.85 \mathrm{MHz}$ \\
\hline Pulse duration $(T)$ & $0.4 \mathrm{~ms}$ \\
\hline Pulse repetition interval $\left(T_{\mathrm{r}}\right)$ & $4 \mathrm{~ms}$ \\
\hline Frequency step size $(\Delta f)$ & $1 \mathrm{kHz}$ \\
\hline Weight factor $(w)$ & 3.6247 \\
\hline Population size & 100 \\
\hline Crossover fraction & 0.8 \\
\hline Migration fraction & 0.2 \\
\hline Mutational fraction & 0.01 \\
\hline
\end{tabular}

Tab. 1. Simulation Parameters.

\begin{tabular}{|c|c|c|c|c|c|c|c|c|c|c|c|c|}
\hline \multirow[t]{2}{*}{ No } & \multicolumn{3}{|c|}{$\begin{array}{c}\text { Frequency } \\
\text { code }\end{array}$} & \multicolumn{3}{|c|}{$\begin{array}{c}\text { Added } \\
\text { phase code }\end{array}$} & \multicolumn{6}{|c|}{ DFPCW-2 } \\
\hline & $1 \mathrm{~F}$ & $2 \mathrm{~F}$ & $3 \mathrm{~F}$ & $1 \mathrm{P}$ & $2 \mathrm{P}$ & $3 \mathrm{P}$ & $1 \mathrm{~F}$ & $2 \mathrm{~F}$ & $3 \mathrm{~F}$ & $1 \mathrm{P}$ & $2 \mathrm{P}$ & $3 \mathrm{P}$ \\
\hline & 10 & 22 & 13 & 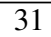 & 31 & $\Omega$ & 14 & 11 & 22 & 16 & 24 & 6 \\
\hline & & 19 & 24 & 1 & 0 & 0 & 19 & 16 & 5 & 8 & 19 & 12 \\
\hline & 22 & 20 & 26 & & 2 & $\overline{0}$ & 22 & 28 & 13 & 12 & 31 & 26 \\
\hline & & 27 & 12 & 1 & 51 & 0 & & 23 & 15 & 14 & 21 & 16 \\
\hline 3 & 4 & 16 & 6 & 1 & 31 & 0 & 7 & & 27 & 12 & 18 & 20 \\
\hline 6 & 16 & 20 & 13 & & ( & 31 & 11 & 20 & 1 & 21 & 5 & 15 \\
\hline 7 & & 11 & 6 & 31 & 0 & 31 & 10 & 0 & 24 & 21 & 17 & 25 \\
\hline 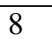 & 13 & 27 & 21 & 1 & 0 & 31 & & 16 & - & 10 & 14 & 5 \\
\hline $\bar{c}$ & & 12 & 22 & 31 & 0 & 2 & & 23 & 15 & 17 & 26 & 20 \\
\hline 10 & 8 & 26 & 10 & & 31 & 3 & & 21 & 13 & 11 & 20 & 14 \\
\hline 11 & 21 & 17 & 8 & & & 0 & 16 & 4 & 11 & 17 & 22 & 22 \\
\hline 12 & 3 & 11 & 22 & 0 & 31 & 0 & 1 & 14 & 21 & 14 & 27 & 9 \\
\hline 13 & 29 & 16 & 2 & 1 & & 3 & 3 & 10 & 17 & 5 & 12 & 10 \\
\hline 14 & 14 & 22 & 11 & 31 & 0 & 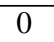 & 22 & 19 & 11 & 11 & 27 & 2 \\
\hline 15 & 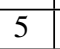 & 24 & 3 & 0 & 0 & 31 & & 12 & 18 & 5 & 25 & 8 \\
\hline 16 & 19 & 7 & 11 & 31 & 31 & 0 & & 26 & 23 & 18 & 22 & 10 \\
\hline 17 & 4 & 31 & 0 & 31 & 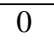 & 31 & & 23 & 26 & 21 & 13 & 5 \\
\hline 18 & 22 & 3 & 1 & - & 3 & 3 & 21 & 11 & 8 & 26 & 16 & 23 \\
\hline 19 & 26 & 14 & 17 & & 0 & 0 & 15 & 8 & 5 & 30 & 0 & 6 \\
\hline 20 & 12 & 8 & 23 & 0 & 0 & 0 & 12 & 19 & I & 7 & 18 & 12 \\
\hline 21 & 17 & 18 & 12 & + & 31 & 0 & 12 & 19 & 30 & 12 & 18 & 25 \\
\hline 22 & 13 & 28 & 16 & 31 & $\Omega$ & 0 & 16 & 13 & 8 & 7 & 6 & 17 \\
\hline 23 & 9 & 14 & 4 & 0 & 0 & 0 & 16 & 20 & 23 & 14 & 14 & 19 \\
\hline 24 & 20 & 6 & 28 & 0 & 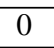 & 0 & & 26 & 19 & 12 & 9 & 19 \\
\hline 25 & 10 & 25 & 20 & $\overline{0}$ & 31 & 31 & & 13 & 24 & 17 & 28 & 1 \\
\hline 26 & 14 & 0 & 10 & 0 & 0 & 31 & 22 & 25 & 15 & 9 & 11 & 5 \\
\hline 27 & 15 & 23 & 31 & 0 & 31 & 31 & 24 & 17 & 3 & 1 & 23 & 31 \\
\hline 28 & 17 & 9 & 12 & 31 & 16 & 0 & 3 & 17 & 14 & 6 & 13 & 8 \\
\hline 29 & 11 & 5 & 24 & 0 & 0 & 31 & 1 & & 0 & 27 & 26 & 10 \\
\hline 30 & 7 & 17 & 22 & 31 & 31 & 31 & 19 & 15 & 10 & 5 & 28 & 12 \\
\hline 31 & 24 & 14 & 19 & 31 & 10 & 31 & 25 & 18 & 17 & 11 & 5 & 8 \\
\hline 32 & 15 & 12 & 25 & 31 & S & 31 & 28 & 14 & 19 & 7 & 11 & \\
\hline
\end{tabular}

Tab. 2. Optimal code sequences of DFCW and DFPCW. The $1 \mathrm{~F}, 2 \mathrm{~F}$, and $3 \mathrm{~F}$ are the frequency code sequences of the three signals, respectively, while the $1 \mathrm{P}, 2 \mathrm{P}$ and $3 \mathrm{P}$ are the phase code sequences.

of the algorithm is when the variation of the fitness function is less than $10^{-6}$ or the optimal individual in the population has been consistent for 50 generations. The radar system we considered here is a high frequency surface wave radar applied to ship detection. The range resolution is about $5 \mathrm{~km}$ corresponding to the total bandwidth, and the maximum range is $600 \mathrm{~km}$ according to the pulse repetition interval.

\subsection{Optimal Waveforms}

The optimal frequency sequences of the DFCW obtained by the algorithm given above are shown in the 2 nd to 4th columns (with the column header of "Frequency code") in Tab. 2. And the ASPs, CPs and the value of fitness function are shown in the 2 nd to 4 th rows (i.e. DFCW) in Tab. 3. After optimization, the value of fitness function of the optimal DFCW reaches 0.6026 . Afterwards, we modulate the initial phase of each pulse using 32-phase discrete phase code on condition that the frequency coding remain constant. With simply changing the phase of each pulse, waveforms can be 


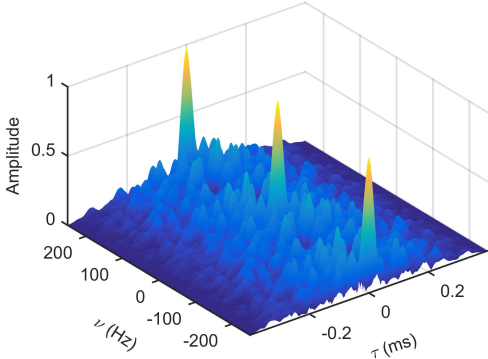

(a)

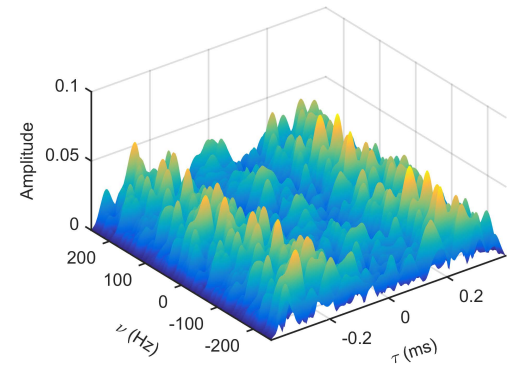

(d)

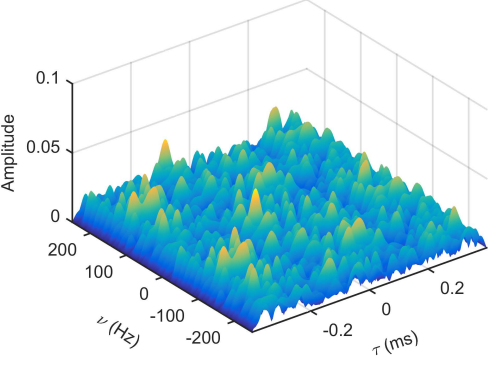

(e)

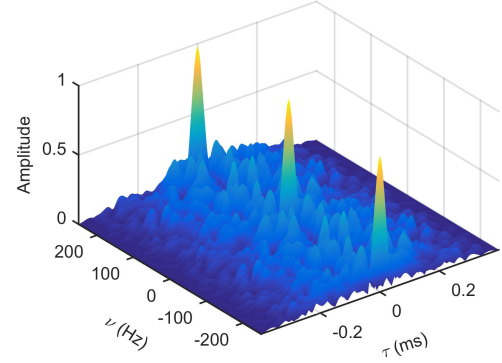

(c)

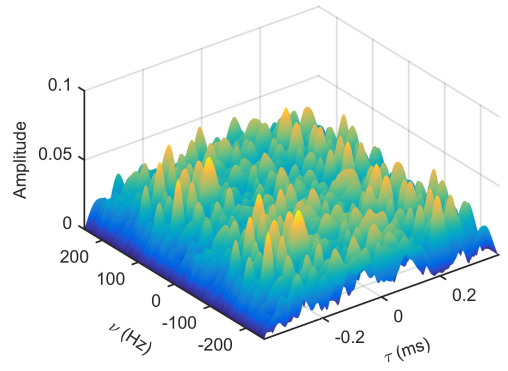

(f)

given in (a), (b) and (c), respectively. (d), (e) and (f) shows the cross ambiguity function of signal 1 and signal 2 , signal 1 and signal 3 , and signal 2 and signal 3 , respectively.

further optimized. The resulting phase coding sequence are given in the 5th to 7th columns (with the column header of "Added phase code") in Tab. 2 and the relevant ASPs as well as CPs the 5th to 7th rows (i.e. DFPCW-1) in Tab. 3, respectively. The same ASP value of the two waveforms validates the uniformity of the ambiguity function of DFPCW-1 and that of DFCW, which matched to (9) we deduced. Clearly, the DFPCW-1 possesses lower CPs than that of the DFCW, along with a reduction by 0.0361 of the fitness value. It is clear evident that the $\mathrm{CP}$ of the optimal DFCW can be further reduced by using phase coding, which implies that DFPCW-1 has better orthogonality than DFCW.

\begin{tabular}{|c|c|c|c|c|c|}
\hline Waveforms & & S1 & S2 & S3 & Fitness \\
\hline \multirow{3}{*}{ DFCW } & S1 & 0.0654 & 0.0353 & 0.0326 & \\
\cline { 2 - 5 } & S2 & 0.0353 & 0.0697 & 0.0255 & 0.6026 \\
\cline { 2 - 5 } & S3 & 0.0326 & 0.0255 & 0.1359 & \\
\hline \multirow{3}{*}{ DFPCW-1 } & S1 & 0.0654 & 0.0308 & 0.0281 & \\
\cline { 2 - 5 } & S2 & 0.0308 & 0.0697 & 0.0226 & 0.5665 \\
\cline { 2 - 5 } & S3 & 0.0281 & 0.0226 & 0.1359 & \\
\hline \multirow{3}{*}{ DFPCW-2 } & S1 & 0.0942 & 0.0294 & 0.0181 & \multirow{3}{*}{0.5425} \\
\cline { 2 - 5 } & $\mathbf{S 2}$ & 0.0294 & 0.0731 & 0.0325 & \\
\cline { 2 - 5 } & $\mathbf{S 3}$ & 0.0181 & 0.0325 & 0.0854 & \\
\hline
\end{tabular}

Tab. 3. ASPs, CPs and fitness values of DFCW and DFPCWs. $\mathrm{S} 1, \mathrm{~S} 2$ and $\mathrm{S} 3$ are the individual signals in a waveform set, respectively. For each waveform, the values on the diagonal of the $3 \times 3$ subtable are the ASP of each signal in the waveform set. The other six values in the subtable are the CPs between two signals.
The above mentioned design method split the optimizing process into two parts, offering advantages such as lower space requirement for solutions and shorter time taken to get the solutions. However, the method may result in local optimization. To avoid this problem, the frequency code sequences and phase code sequences are optimized simultaneously and thus achieved the more desirable waveforms. The resulting optimal coding sequences are given in 8th to 13th in Tab. 2, and the ASPs, CPs and fitness value after optimization are shown in the 8 th to 10 th rows (i.e. DFPCW-2) in Tab. 3.

The optimal designed DFPCW is shown in Fig. 3. In Fig. 3(a), 3(b), and 3(c), although the sidelobe peaks are clearly observable, the "thumbtack" feature of the mainlobe is evident. The narrow shape of the mainlobe indicates that the DFPCW possesses good resolution both in time-delay and Doppler shift. The grating lobes at Doppler frequency of about $\pm 250 \mathrm{~Hz}$ are negligible, since in practice no artificial vehicle can reach those high velocities. The cross ambiguity peaks in Fig. 3(d), 3(e), and 3(f) are randomly distributed due to the random property of the frequency and phase code, however, most of the peaks are less than 0.05 demonstrating that the mutual interference between DFPCWs is low.

\subsection{Comparison with Existing Waveforms}

Comparisons with existing waveforms are also given using the parameters listed in Tab. 1. In Fig. 4, we compare 


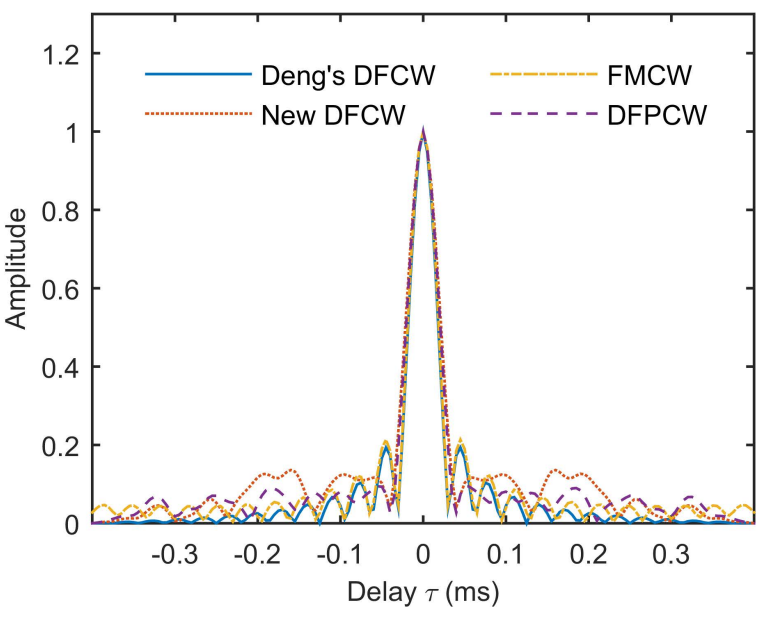

(a)

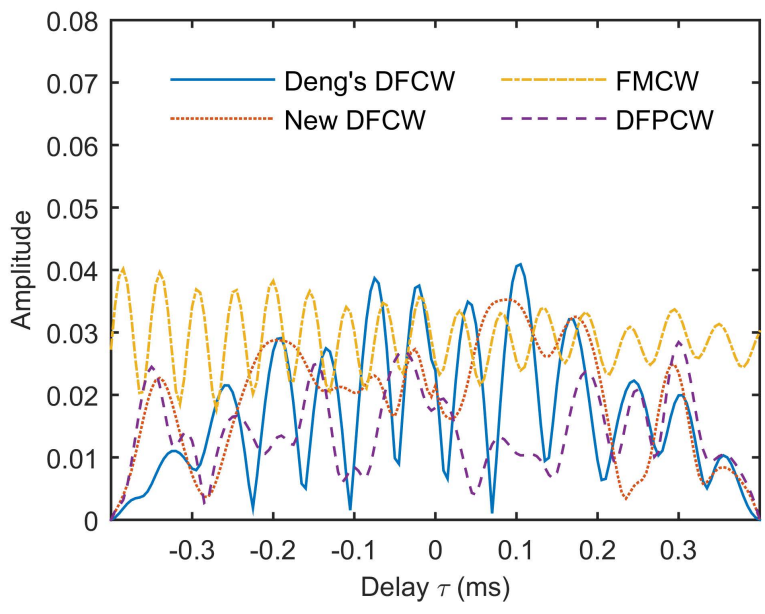

(b)

Fig. 4. Comparisons of the designed DFPCW with existing waveforms. (a) is the autocorrelation of the four waveforms, where the ASPs of Deng's DFCW, New DFCW, FMCW and DFPCW are $0.1933,0.1359,0.2134$ and 0.0942 , respectively. (b) is the cross-correlation of the four waveforms, where the CPs of Deng's DFCW, New DFCW, FMCW and DFPCW are 0.040 9, 0.0353 , 0.0402 and 0.0285 , respectively.

the DFPCW with the Deng's DFCW, New DFCW and frequency modulated continuous wave (FMCW) in the aspects of auto-correlation function and cross-correlation function. As Deng's DFCW considers optimal a continuous wave, we modified this waveform in our optimal criteria obtaining the new DFCW (a pulse train waveform utilizing frequency diversity). The FMCW set employed here contains two waveforms having opposite frequency modulation [20].

Figure 4(a) demonstrates that, the mainlobe of those four waveforms are nearly the same, however, the ASP of DFPCW is dramatically lower than that of the other waveforms. From Fig. 4(b), we find that the Deng's DFCWs have the highest $\mathrm{CP}$, which may due to the changed simulation condition (including pulse duration, bandwidth, etc.) in this paper. However, the New DFCW obtained by employing genetic algorithm with the simulation condition as shown in Tab. 1 presents better orthogonality. Obviously, the DFPCW has lowest peak value, compared with the new designed optimal DFCW and FMCW.

\section{Conclusions}

In this paper, a novel DFPCW consisting of both discrete frequency code and initial phase code is proposed, followed by boosting the orthogonality of waveforms benefits from the diversity characteristics. By changing the initial phase of each pulse, the DFPCW achieved lower CP than that of the DFCW, on the premise that they have the same autocorrelation features. Furthermore, the fitness value drove lower to 0.5425 when achieving both the frequency optimization and the phase optimization simultaneously. Both the two results show that DFPCW is superior to DFCW. The Comparison with widely employed FMCW also validate the superiorities of the designed waveform. In the future, a waveform contains more diversities rather than only frequency diversity and phase diversity employed here will be investigated.

\section{Acknowledgments}

This work is supported in part by the National Natural Science Foundation of China under Grant No. 61571159, and the Public Science and Technology Research Funds Projects of Ocean under Grant No. 201505002.

\section{References}

[1] FISHLER,E., HAIMOVICH, A., BLUM, R., et al. MIMO radar: An idea whose time has come. In Proceedings of the 2004 IEEE Radar Conference. Philadelphia (USA), 2004, p. 71-78. DOI: $10.1109 /$ NRC.2004.1316398

[2] FISHLER, E., HAIMOVICH, A., BLUM, R. S., et al. Spatial diversity in radars-models and detection performance. IEEE Transactions on Signal Processing, 2006, vol. 54, no. 3, p. 823-838. DOI: 10.1109/TSP.2005.862813

[3] LI, J., STOICA, P. MIMO Radar Signal Processing. New York (USA): John Wiley \& Sons, Inc., 2008. ISBN: 978-0-470-17898-0

[4] FRIEDLANDER, B. On the relationship between MIMO and SIMO radars. IEEE Transactions on Signal Processing, 2009, vol. 57, no. 1, p. 394-398. DOI: 10.1109/TSP.2008.2007106

[5] SAMMARTINO, P. F., BAKER, C. J., GRIFFITHS, H. D. Frequency diverse MIMO techniques for radar. IEEE Transactions on Aerospace and Electronic Systems, 2013, vol. 49, no. 1, p. 201-222. DOI: 10.1109/TAES.2013.6404099

[6] SUN, H., GAO, C., TEH, K. C. Performance evaluation of practical MIMO radar waveforms. In Proceedings of the 2016 IEEE Radar Conference. Philadelphia (USA), 2016, p. 1-6. DOI: 10.1109/RADAR.2016.7485172

[7] MAJUMDER, U., BELL, M. R., RANGASWAMY, M. Design and analysis of radar waveforms achieving transmit and receive orthogonality. IEEE Transactions on Aerospace and Electronic Systems, 2016, vol. 52, no. 3, p. 1056-1066. DOI: 10.1109/TAES.2016.140248

[8] SUN, H., BRIGUI, F., LESTURGIE, M. Analysis and comparison of MIMO radar waveforms. In Proceedings of the 2014 
International Radar Conference. Lille (France), 2014, p. 1-6. DOI: 10.1109/RADAR.2014.7060251

[9] DENG, H. Polyphase code design for orthogonal netted radar systems. IEEE Transactions on Signal Processing, 2004, vol. 52, no. 11 , p. 3126-3135. DOI: 10.1109/TSP.2004.836530

[10] REDDY, B. R., KUMARI, M. U. Polyphase orthogonal waveform using modified particle swarm optimization algorithm for MIMO radar. In Proceedings of the 2012 IEEE International Conference on Signal Processing, Computing and Control (ISPCC). Waknaghat Solan (India), 2012, p. 1-6. DOI: 10.1109/ISPCC.2012.6224367

[11] DENG, H. Discrete frequency-coding waveform design for netted radar systems. IEEE Signal Processing Letters, 2004, vol. 11, no. 2, p. 179-182. DOI: 10.1109/LSP.2003.821693

[12] REDDY, B. R., KUMARI, M. U. Optimization of discrete frequency coding waveform for MIMO radar using modified ant colony optimization algorithm. In Proceedings of the 2014 First International Conference on Networks and Soft Computing (ICNSC). Guntur (India), 2014, p. 15-19. DOI: 10.1109/CNSC.2014.6906667

[13] YA-LI, P., JIN, Y. Chaos based orthogonal discrete frequency coding waveform design. In Proceedings of the 2013 IEEE China Summit and International Conference on Signal and Information Processing (ChinaSIP). Beijing (China), 2013, p. 30-33. DOI: 10.1109/ChinaSIP.2013.6625291

[14] ZHANG, Y., WANG, J. Improved design of DFCW for MIMO radar. Electronics Letters, 2009, vol. 45, no. 5, p. 285-286. DOI: 10.1049/el:20093665

[15] MEHANY, W., JIAO, L. Improved design of orthogonal discrete frequency-coding waveform based on modified genetic algorithm for MIMO-SAR. In Proceedings of the 2014 IEEE International Conference on Signal Processing, Communications and Computing (ICSPCC). Guilin (China), 2014, p. 341-345. DOI: 10.1109/ICSPCC.2014.6986211

[16] LIU, B. Orthogonal discrete frequency-coding waveform set design with minimized autocorrelation sidelobes. IEEE Transactions on Aerospace and Electronic Systems, 2009, vol. 45, no. 4, p. 1650-1657. DOI: 10.1109/TAES.2009.5310326

[17] GAO, C., TEH, K. C., LIU, A. Orthogonal frequency diversity waveform with range-Doppler optimization for MIMO radar. IEEE Signal Processing Letters, 2014, vol. 21, no. 10, p. 1201-1205. DOI: 10.1109/LSP.2014.2329944

[18] GAO, C., TEH, K. C., LIU, A. Frequency coding waveform with segment LFM. In Proceedings of the 2015 IEEE 5th Asia-Pacific Conference on Synthetic Aperture Radar (APSAR). Singapore, 2015, p.507--510. DOI: 10.1109/APSAR.2015.7306260
[19] LEVANON, N., MOZESON, E. Radar Signals. New Jersey (USA): John Wiley \& Sons, Inc., 2004. ISBN: 0-471-47378-2

[20] WANG, W. Q. MIMO SAR chirp modulation diversity waveform design. IEEE Geoscience and Remote Sensing Letters, 2014, vol. 11, no. 9, p. 1644-1648. DOI: 10.1109/LGRS.2014.2303974

\section{About the Authors...}

Guanghong CHANG was born in 1990 in Tai' an, China. He received the B.S. degree in automation from Qingdao University of Science and Technology, Qingdao, China, in 2011 and the M.S. degree in control theory and control engineering from Ocean University of China, Qingdao, China, in 2014. $\mathrm{He}$ is currently working toward the Ph.D. degree in information and communication engineering at Harbin Institute of Technology, Harbin, China. His past and present research involves the inversion of ocean wave spectrum from highfrequency surface wave radar (HFSWR) spectra, measurement of ocean surface current using shipborne HF radar and, more recently, design of transmitting waveform and analyses of multiple-input-multiple-output (MIMO) HF radar system performance.

Xiaoxi YU (corresponding author) was born in 1993. She received her BEng degree in electrical and electronics engineering from University of Bristol, UK, and is currently studying for the Msc degree in wireless communications and signal processing at University of Bristol. Her research interests include MIMO theory and applications, electromagnetic compatibility and microwave communications.

Changjun YU was born in Harbin, China. He received the B.S. degree in electronic engineering from Harbin Institute of Shipbuilding Engineering, Harbin, China, in 1984 and the M.S. and Ph.D. degrees in information and communication engineering from Harbin Engineering University, Harbin, China, in 1990 and 2010, respectively. He is a Researcher in the School of Information and Electrical Engineering, Harbin Institute of Technology at Weihai, Weihai, China. His research interests involve high-frequency surface wave radar (HFSWR) system design and analysis, target detection, estimation and tracing, and ionosphere remote sensing. 